\title{
Sistema de Medição de Desempenho na Graduação em Engenharia: Aplicação em um Curso de Engenharia de Produção
}

\author{
Víctor Cussiol Macul, André Bain, Eduardo de Senzi Zancul* \\ Departamento de Engenharia de Produção da Escola Politécnica da Universidade de São Paulo \\ * Autor para correspondência: ezancul@usp.br
}

\begin{abstract}
RESUMO
A medição do desempenho é uma importante ferramenta para a gestão da educação em instituições de ensino superior. Nelas, esse tipo de mensuração é especialmente interessante, pois se revela útil para a gestão da universidade, sobretudo no que diz respeito ao acompanhamento de resultados e metas e ao atendimento de demandas externas. Diversos stakeholders (gestores acadêmicos, coordenadores de curso, instituições de governo, entidades de fomento, dentre outros) buscam cada vez mais clareza sobre o desempenho das universidades para direcionar políticas e investimentos. Nesse contexto, o presente trabalho foi desenvolvido com o objetivo de fornecer ao Departamento de Engenharia de Produção da Escola Politécnica da Universidade de São Paulo uma proposta de ferramenta que cumpra a demanda de medição de desempenho de seu curso de graduação. Por meio da aplicação de metodologia específica, foi desenvolvida uma proposta de um conjunto de indicadores de desempenho para graduação em Engenharia. Como principais resultados, destacam-se: a definição de um Sistema de Medição de Desempenho (SMD) aplicável na educação superior; a identificação de requisitos operacionais para indicadores; a seleção de indicadores de desempenho específicos; a definição de um formato adequado de ficha de indicadores; e a confecção de um painel de gestão (dashboard). Nesse sentido, o presente trabalho pode servir de referência para a elaboração de sistemas semelhantes de medição de desempenho em outros cursos de graduação em Engenharia, tendo em vista a melhoria contínua da qualidade do ensino oferecido.
\end{abstract}

Palavras-Chave: Sistema de Medição de Desempenho (SMD); Indicadores de Desempenho; Balanced Scorecard (BSC); Ensino Superior; Educação Superior.

\begin{abstract}
Performance measurement is an essential tool for the management of higher education institutions. This kind of measurement is especially interesting in these institutions because it proves useful for the management of the university itself, especially about the monitoring of results and targets and the fulfillment of external demands. Many stakeholders (academic managers, course coordinators, government institutions, development agencies, among others), increasingly seek clarity on the performance of public universities to direct policies and investments. In this context, the present work was developed to provide the Departamento de Engenharia de Produção da Escola Politécnica da Universidade de São Paulo with the proposal of a tool that meets the performance measurement demand of its undergraduate program. Through the application of specific methodology, a proposal of a set of key performance indicators (KPIs) for Engineering major was developed. The primary results are the definition of a performance measurement system (PMS) with a format applicable in higher education; the list of operational requirements for indicators; the selection of performance indicators; the definition of a suitable model of indicator form; and the organization of the KPIs in a dashboard. In this sense, this work can serve as a reference for the elaboration of similar performance measurement systems in other Engineering majors, with the goal of continuously improving the quality of the education offered.
\end{abstract}

Keywords: Performance Measurement System (PMS); Key Performance Indicators (KPIs); Balanced Scorecard (BSC); Higher Education; College Education.

\section{Introdução}

A expansão do acesso à educação superior envolve investimentos e gastos consideráveis da sociedade, tanto em instituições públicas como em instituições privadas - com ou sem fins lucrativos. Em busca de maior transparência, eficiência e 
melhores resultados, sistemas que avaliam os resultados da educação superior vêm sendo criados e aprimorados.

Nesse contexto, ganha força o conceito de accountability (ou prestação de contas, em tradução livre dos autores), que consiste na tendência, em diversos países, ao emprego de sistemas de medição de desempenho que possibilitem a avaliação e a comparação de instituições de ensino superior (IES) (ALEXANDER, 2000).

Essa tendência nem sempre é aceita com facilidade, por alguns motivos, tais como a necessidade de se combinar e balancear a visão de gestão, geralmente mais focada em indicadores de eficiência, com a visão acadêmica, mais focada em medidas de desenvolvimento pessoal e da qualidade de ensino. Ainda assim, é possível unir essas duas visões para formar Sistemas de Medição de Desempenho (SMDs) que abranjam de forma interessante tanto aspectos de produtividade quanto fatores de qualidade.

Um obstáculo comum à utilização de SMDs em educação superior é a necessidade de uma complexa coleta e tratamento de dados. Em geral, isso se dá pela falta de uma base de dados estruturada sobre universidades em cada país. Faltam padrões nacionais de desempenho que contribuam para a comparação e a validação dos dados obtidos (THOMSON REUTERS, 2010). Além de indicadores amplos baseados em dados públicos, um SMD para educação superior pode envolver dados mais específicos e detalhados de cada instituição e de seus cursos, que podem estar armazenados em diversos sistemas de informação. Sendo assim, muitas vezes a tarefa de implantar o SMD parece complicada demais e não avança.

Para enfrentar essa situação, pode-se conceber uma aplicação piloto, demostrando a sua utilidade em uma situação específica. Considera-se aqui um SMD que possa apoiar o acompanhamento e a melhoria do ensino e da gestão de cursos de graduação em Engenharia, ou seja, a unidade de análise e de atuação é de cada curso, com indicadores mais detalhados e significativos, que vão além dos requisitos da regulamentação e avaliação oficial de cursos. Como citado no relatório da Thomson Reuters de 2010 (p. 4): "Instituições (de ensino superior) não podem contar apenas com a avaliação de seus pares e com sua reputação, elas devem ser capazes de medir quantitativamente (seu desempenho) para melhorar seu desempenho".

Nesse contexto, este artigo tem como objetivo apresentar uma proposta de SMD para cursos de engenharia. A proposta é aplicada no curso de Engenharia de Produção da Escola Politécnica da Universidade de São Paulo (EP-USP).

\section{Sistemas de Medição de Desempenho}

Para Spinola e Pessôa (1997, p. 99), "A informação é uma ferramenta poderosa para uma organização, pois através dela pode-se ter um domínio dos diversos parâmetros que regem a sua dinâmica". Sendo assim, a gestão do desempenho precisa, como pré-requisito para ser eficaz, de um conjunto de informações que possibilite a tomada de uma decisão embasada.

Araujo (2001, p. 77) argumenta que "a gestão do desempenho deve proporcionar uma ligação sistemática entre a estratégia organizacional, os recursos e os processos, de uma forma estruturada, para a obtenção de melhorias contínuas, de modo que todos os envolvidos entendam onde se está e aonde se quer chegar para atender os interesses dos stakeholders".

Podem-se destacar três dimensões necessárias para a elaboração de um SMD (ARAUJO, 2001): (1) atenção a diferentes stakeholders (atores); (2) possibilidade de comparação e "melhorias contínuas"; e (3) ligação entre os indicadores, estratégia organizacional, recursos e processos. Dessa forma, garante-se que as métricas geradas cumpram seus objetivos de mostrar com clareza e precisão os números mais relevantes.

Um indicador de desempenho deve atender a uma série de requisitos operacionais (MACHADO, 2001; FRANCISCHINI, 2014; BORGES \& CARVALHO, 2011): (1) ser quantificável e mesurável, indicado por medidas numéricas; (2) ser compreensível e mostrar com clareza as informações-chave; (3) ser ajustável e 
controlável, referir-se a um processo passível de ajuste, buscando a melhoria contínua; (4) ser sensível às variações do processo, indicar com precisão a situação real, correlacionar as decisões operacionais e resultados; (5) ser rastreável, assinalar qual o problema e qual a sua raiz, apontando a direção da melhoria; (6) ser objetivo e completo, referir diretamente aquilo que se quer medir com completude; (7) relacionar-se diretamente com a estratégia da empresa e ser um indicador-chave; (8) ser facilmente atualizável na periodicidade adequada; e (9) ser correto, preciso e válido, ao garantir que os dados sejam obtidos e processados apropriadamente, gerando números que representam de maneira significativa a situação real.

Em um SMD, indicadores de desempenho têm o papel de "quantificar o desempenho do objeto de estudo" (KIYAN, 2001, p. 26), e o conjunto de indicadores forma o SMD. Rosa et al. (1995, p. 522) afirmam que parâmetros de desempenho "representam um conjunto de informações necessárias para que as equipes gerenciais possam administrar a competitividade do sistema organizacional. Funcionam como instrumentos, cujos mostradores são representados pelos indicadores específicos".

Nesse sentido, o chamado Balanced Scorecard (BSG) visa a avaliar instituições de maneira balanceada, sem colocar peso demais em um tipo específico de medida, como ocorria anteriormente, com as medidas contábeis-financeiras. Além da perspectiva financeira, no BSC são exploradas as perspectivas dos clientes, dos processos de negócio internos, de aprendizado e crescimento. Um Balanced Scorecard bem construído pode retratar a situação de uma organização, dada a relação de causa e efeito entre as perspectivas (KAPLAN \& NORTON, 1996).

Mais do que apenas fornecer um guia de como criar um conjunto de indicadores amplo e eficiente, o BSC desempenha outra função importante de forma efetiva: ele ajuda a converter a visão e a estratégia de mais alto nível da instituição em ações concretas para atingi-las. Podem-se destacar, para cada perspectiva do BSC, quatro elementos essenciais: (1) Objetivos do gestor da organização, derivados da estratégia e visão; (2) Medidas e parâmetros a serem mensurados para se rastrear o progresso em relação aos objetivos traçados; (3) Metas, valor de referência que se deseja obter para um determinado indicador em certo período de tempo; e (4) Iniciativas, planos de ação necessários para se atingir a meta traçada para cada indicador (KAPLAN \& NORTON, 1996).

A premissa considerada neste texto é a de que a base conceitual de SMDs é útil e pode ser adaptada para ser aplicada em instituições de ensino superior para atender a demanda crescente de acompanhamento de resultados. Na próxima seção é discutida a aplicação de indicadores na educação superior.

\section{Indicadores em Educação Superior}

Por causa de suas características diferentes de uma empresa tradicional e suas relações complexas com a sociedade, as universidades precisam de um conjunto de indicadores adaptado.

Três formas de medição ganham destaque na literatura: a primeira é resultado de um estudo da Educational Commission dos Estados Unidos, que aponta os indicadores propostos para avaliar instituições de ensino superior em dez estados americanos. Os indicadores coletados são divididos em oito categorias: (1) entradas instrucionais, com indicadores como pontuação dos calouros no processo seletivo e número de alunos em recuperação; (2) processos instrucionais, com distribuição de créditos por disciplina e período; (3) resultados instrucionais, com número de concluintes, colocações no mercado de trabalho e satisfação do aluno com o curso; (4) eficiência e produtividade, com indicadores como custo dos programas e carga de trabalho dos professores (5) condição do ativo, com percentual de financiamento familiar e medidas de atividades de pesquisa patrocinadas externamente; (6) diversidade / acesso / equidade, com estatísticas de conclusão por raça e gênero; (7) ligação com a educação primária e secundária (K12), com indicadores como volume e desempenho de alunos 
de transferência entre programas de dois anos (two-year college) para programas mais longos; e (8) relação com as necessidades do Estado, com medições para a relação dos programas com as necessidades de emprego (EWELL, 1994). Ainda que amplamente utilizados, esses indicadores são primordialmente históricos, limitados em poder preditivo.

A segunda é a adaptação do BSC feita, por exemplo, em países como Escócia, Taiwan e Malásia (MARTIN et al., 2011; CHEN et al., 2006). Nesse caso, é comum adaptar a perspectiva do cliente do BSC para a perspectiva do stakeholder, incluindo ex, atuais e futuros alunos, sociedade e governo, ao passo que as outras perspectivas se mantêm iguais às do BSC original.

Outra forma de destaque é a proposta pelo Malcom Baldrige National Quality Program Award, conforme descrito por Beard (2009). Nesse caso, também se faz uma adaptação do BSC, mas de maneira mais extensa, incluindo em sua forma final seis categorias de desempenho. São elas: resultados de aprendizado dos estudantes; resultados focados nos estudantes e demais stakeholders, como candidatos inscritos no vestibular e ex-alunos; resultados financeiros e de orçamento; resultados de professores e staff; resultados de eficiência organizacional interna; resultados de governança e responsabilidade social.

\section{Metodologia}

Para a realização deste trabalho, algumas etapas principais foram cumpridas. Primeiramente, foi feita uma revisão da literatura com foco em práticas estabelecidas para a criação e organização de indicadores de desempenho, inclusive relacionados com educação superior. Em seguida, com base na literatura, foram definidos os passos para a elaboração de um sistema de indicadores de desempenho, apresentados na Tabela 1. Por fim, foi aplicada a perspectiva do BSC adaptado para este SMD. Simultaneamente à elaboração de indicadores, ocorre o processo de conferir se eles cumprem os requisitos operacionais estudados na literatura. Os indicadores que passaram pelo processo de seleção feito na etapa de anterior - e cujos dados puderam ser obtidos - foram então submetidos ao processo de coleta de dados para o curso de Engenharia de Produção da Escola Politécnica da Universidade de São Paulo (EP-USP). Para essa etapa, diversas fontes foram consultadas.

Para dados de captação de alunos, a fonte mais utilizada foi a Fundação Universitária para o Vestibular (Fuvest, 2015), que divulga os dados organizados por curso e gênero de alunos inscritos e convocados. Para dados de formação de alunos, as fontes mais consultadas foram a Seção de Alunos da EP-USP e a secretaria de graduação do Departamento de Engenharia de Produção, bem como os sistemas de gestão da graduação empregados na USP, como o JúpiterWeb.

De acordo com suas respectivas fórmulas, os valores para os indicadores são calculados, incluindo tanto os dados referentes ao curso de Engenharia de Produção quanto aqueles referentes aos benchmarks, ou seja, referências utilizadas para fins de comparação, como outros cursos da EP-USP e outros cursos USP de Engenharia de Produção (Escola de Engenharia de Lorena e Escola de Engenharia de São Carlos).

Por fim, com toda a informação necessária coletada e processada, é realizada a documentação dos indicadores no formato de fichas com o apoio de uma planilha eletrônica ( $M S$ Excel), para facilitar a sua compreensão e posterior atualização. As fichas não incluem gráficos, já que esses são apresentados por meio de um painel de gestão e navegação (chamado de dashboard), construído com o apoio das ferramentas de apresentação eletrônica combinada ao DataHero®, software on-line cuja função é justamente organizar gráficos e dashboards.

Vale ressaltar que o passo a passo apresentado na Tabela 1 esteve sujeito a diversas verificações intermediárias com o representante da Comissão do Curso (CoG) envolvido com o trabalho, sempre com o intuito de garantir que o trabalho desenvolvido estivesse de acordo com as prioridades e demandas da CoC. 


\begin{tabular}{|c|c|c|}
\hline Passo & Atividade & Fonte \\
\hline 1 & $\begin{array}{l}\text { Identificar os principais stakeholders para a } \\
\text { instituição }\end{array}$ & Borges e Carvalho (2011) \\
\hline 2 & $\begin{array}{l}\text { Estruturar e priorizar os objetivos organizacio- } \\
\text { nais / objetivos do gestor tendo em vista as } \\
\text { prioridades dos stakeholders }\end{array}$ & Borges e Carvalho (2011) \\
\hline 3 & $\begin{array}{l}\text { Determinar os Critérios Relevantes de } \\
\text { Interpretação (CRIs), ou seja, variáveis ou atri- } \\
\text { butos mais importantes que mostram como o } \\
\text { objetivo deve ser medido }\end{array}$ & Francischini (2014) \\
\hline 4 & $\begin{array}{l}\text { Desdobrar os CRIs em indicadores de desem- } \\
\text { penho que sirvam como conjunto de métricas } \\
\text { que traduzam e esclareçam os CRIs }\end{array}$ & Francischini (2014) \\
\hline 5 & $\begin{array}{l}\text { Determinar a base de comparação entre o de- } \\
\text { sempenho almejado e o real, por meio de me- } \\
\text { tas, benchmarks ou tendências históricas }\end{array}$ & Slack e Lewis (2009) \\
\hline 6 & $\begin{array}{l}\text { Coletar os dados necessários para a mensura- } \\
\text { ção, garantindo que as fontes de informação } \\
\text { estejam disponíveis e acessíveis }\end{array}$ & Sink e Tuttle (1989) \\
\hline 7 & $\begin{array}{l}\text { Decidir como processar os dados e como apre- } \\
\text { sentar as informações obtidas }\end{array}$ & Sink e Tuttle (1989) \\
\hline
\end{tabular}

Tabela 1 - Passos para a elaboração de um sistema de indicadores de desempenho.

\section{Análise dos Resultados e Discussões}

Foram desenvolvidos indicadores de desempenho relacionados com cinco perspectivas de atuação: financeira; de stakeholders (dividida em captação de alunos, formação de alunos e atuação profissional de alunos); de processos de negócios internos; de aprendizado e crescimento; e de responsabilidade social. As Tabelas 2 a 7 apresentam a lista completa de indicadores por perspectiva, classificados como "disponível", quando seus números puderam ser coletados de forma organizada, ou "proposto", em caso contrário.

Muitas vezes, indicadores foram deixados de lado pois não cumpriam o requisito 3 (ser ajustável e controlável cf. pp. 32-33), como, por exemplo, a razão entre número de alunos e número de professores e funcionários. As ações administrativas sobre tais indicadores não cabem ao departamento de Engenharia de Produção, mas, sim, a outras esferas, como a unidade (Escola Politécnica) ou a Pró-Reitoria de Graduação (PRG).

Para o quinto passo (estabelecer metas e comparações), ficou definido que metas ainda não se aplicavam em um primeiro momento, uma vez que o SMD ainda era novo e metas deveriam ser discutidas e acordadas com os professores e demais envolvidos. Sendo assim, o objetivo foi estabelecer comparações (benchmarks) para os indicadores. Um exemplo de ficha de indicador é apresentado na Figura 1. As fichas de indicadores foram compiladas em um arquivo e disponibilizadas para os professores envolvidos.

A Figura 2 apresenta o dashboard desenvolvido para a apresentação dos gráficos. A utilização da ferramenta DataHero ${ }^{\circledR}$ permitiu um alto nível de interatividade, uma vez que, passando o cursor em cima de cada uma dessas linhas, aparece uma caixa informativa com o nome da série de dados e o valor 


\section{Indicadores de Desempenho \\ Custo total por estudante de graduação \\ Porcentagem da receita gasta com treinamentos e consultorias destinadas ao ensino}

Tabela 2 - Perspectiva Financeira.

\section{Status}

Proposto

Proposto

\section{Status}

Disponível

Disponível

Disponível

Disponível

Disponível

Disponível

Proposto

Proposto

Proposto

Tabela 3 - Perspectiva stakeholders - Captação de alunos.

\section{Indicadores de Desempenho \\ Alunos em duplo diploma no exterior \\ Alunos em aproveitamento de créditos no exterior \\ Alunos estrangeiros em duplo diploma no curso \\ Alunos estrangeiros em aproveitamento de créditos no curso}

\section{Status}

Disponível

Disponível

Disponível

Disponível 


\begin{tabular}{|c|c|}
\hline Estágio por setor da economia - por ano & Disponível \\
\hline Estágio por setor da economia - agregado & Disponível \\
\hline $\begin{array}{l}\text { Estágio em empresa consolidada ou nova - por } \\
\text { ano }\end{array}$ & Disponível \\
\hline $\begin{array}{l}\text { Percentual de satisfação em avaliações } \\
\text { institucionais }\end{array}$ & Proposto \\
\hline $\begin{array}{l}\text { Percentual de satisfação em avaliações } \\
\text { estudantis }\end{array}$ & Proposto \\
\hline $\begin{array}{l}\text { Percentual de premiações de TF dadas a } \\
\text { mulheres }\end{array}$ & Disponível \\
\hline $\begin{array}{l}\text { Percentual de premiações de TF dadas a } \\
\text { alunos vindos de escola pública }\end{array}$ & Proposto \\
\hline Número de alunos envolvidos em pesquisa & Proposto \\
\hline $\begin{array}{l}\text { Número de alunos envolvidos em outras } \\
\text { atividades extracurriculares }\end{array}$ & Proposto \\
\hline Semestres até a graduação - por safra & Disponível \\
\hline Percentual de evasão - por safra & Disponível \\
\hline $\begin{array}{l}\text { Número de premiações de alunos de } \\
\text { graduação }\end{array}$ & Proposto \\
\hline
\end{tabular}

Tabela 4 - Perspectiva stakeholders - Formação de alunos.

\begin{tabular}{|l|l|}
\hline Indicadores de Desempenho & Status \\
\hline $\begin{array}{l}\text { Taxa de empregabilidade no primeiro ano de } \\
\text { graduado }\end{array}$ & Proposto \\
\hline Salário médio no primeiro ano de graduado & Proposto \\
\hline $\begin{array}{l}\text { Percentual de satisfação na primeira atividade } \\
\text { profissional }\end{array}$ & Proposto \\
\hline Avaliação no Guia do Estudante Abril & Disponível \\
\hline Posição no Ranking Folha de Universidades & Disponível \\
\hline Empresas abertas por ano por ex-alunos & Proposto \\
\hline
\end{tabular}


Total de ex-alunos em posição de diretoria por ano

\section{Proposto}

Tabela 5 - Perspectiva stakeholders - Atuação profissional.

\author{
Indicadores de Desempenho \\ Atividades de remediação (contra desistência) \\ e sua efetividade \\ Número de patentes, marcas, propriedades \\ intelectuais, spinoffs gerados na graduação \\ Comparação de valor com os pares: tamanho \\ das turmas
}

Tabela 6 - Perspectiva de processos de negócios internos.

\section{Status}

Proposto

Proposto

Proposto

\section{Status}

Proposto

\section{Proposto}

Proposto

Proposto

Tabela 7 - Perspectiva de responsabilidade social.

no ponto mais próximo. Também é possível filtrar quais pontos no eixo $\mathrm{x}$ (anos, nesse caso) e quais séries de dados são incluídos no gráfico. No entanto, essa ferramenta também possui pontos fracos, como a necessidade de estar conectada à internet, a mensalidade para acesso aos recursos completos e a falta da possibilidade de criar gráficos que sejam editáveis apenas para a visualização - quer dizer, quando se remove ou adiciona algo a um gráfico, isso é automaticamente salvo no arquivo original. O SMD criado também pode ser replicado e adaptado. Ele apresenta um nível considerável de aplicabilidade.
Para melhorar o SMD proposto, buscando uma parcela maior de seus benefícios potenciais, algumas ações são necessárias: a primeira é designar um responsável por sua manutenção e atualização. Seria preciso, também, desenvolver uma cultura organizacional que valorize a coleta sistemática e a estruturação de dados de desempenho como parte essencial da gestão da universidade. Isso torna mais provável que se cumpram as etapas necessárias para a geração e a captura dos dados necessários à elaboração dos indicadores propostos e não desenvolvidos neste trabalho. 


\begin{tabular}{|c|c|c|c|c|c|c|c|c|c|}
\hline Indicador & \multicolumn{5}{|c|}{ Distribuição dos estágios por setor da economia } & & & & \\
\hline \multirow{2}{*}{ Definiçãol 0 que mede } & \multicolumn{5}{|c|}{$\begin{array}{l}\text { Tipos de empresas em que os alunos do PRO fazem seus estágios - evolução } \\
\text { histórica }\end{array}$} & & & & \\
\hline & \multicolumn{5}{|c|}{$\begin{array}{l}\text { Compreender a demanda do mercado por alunos do PRO e o interesse dos } \\
\text { alunos do departamento em cada tipo de empresa, analisar tendências }\end{array}$} & & & & \\
\hline $\begin{array}{l}\text { Objetivol Para que medir? } \\
\text { Perspectiva no BSC }\end{array}$ & \multicolumn{5}{|c|}{ Cliente [stakeholder: alunos] } & & & & \\
\hline \multicolumn{6}{|l|}{ Dados } & & & & \\
\hline \multirow{2}{*}{$\begin{array}{l}\text { Unidade } \\
\text { Fonte de dados }\end{array}$} & \multicolumn{5}{|c|}{ Alunos por setor da economia } & & & & \\
\hline & \multicolumn{5}{|c|}{ Secretaria do PRO } & & & & \\
\hline $\begin{array}{l}\text { Forma de Cálculo } \\
\text { Apresentação dos dados: }\end{array}$ & \multicolumn{5}{|c|}{$\begin{array}{l}\text { Contagem do número de entradas por tipo de empresa a cada ano - entradas } \\
\text { repetidas são contadas, se em anos diferentes }\end{array}$} & & & & \\
\hline Data do Registro & $\begin{array}{l}\text { Dados } \\
\text { referentes a }\end{array}$ & Financeiras & Indústria & Consultoria & $\begin{array}{l}\text { TI, Logística, } \\
\text { Engenharia }\end{array}$ & Dutros & Educação & Comércio & Total \\
\hline 23-02-15 & 2008 & $27,1 \%$ & $14,6 \%$ & $25,0 \%$ & $6,3 \%$ & $4,2 \%$ & $12,5 \%$ & $10,4 \%$ & $100,0 \%$ \\
\hline $23-02-15$ & 2009 & $26,4 \%$ & $34,0 \%$ & $9,4 \%$ & $15,1 \%$ & $1,9 \%$ & $7,5 \%$ & $5,7 \%$ & $100,0 \%$ \\
\hline 23-02-15 & 2010 & $46,2 \%$ & $20.0 \%$ & $12,3 \%$ & $8.5 \%$ & $6,2 \%$ & $5,4 \%$ & $1,5 \%$ & $100,0 \%$ \\
\hline 23-02-15 & 2011 & $40,8 \%$ & $18,3 \%$ & $23,9 \%$ & $5,6 \%$ & $4,2 \%$ & $7,0 \%$ & $-\%$ & $100,0 \%$ \\
\hline 23-02-15 & 2012 & $32,8 \%$ & $21,3 \%$ & $26,2 \%$ & $8.2 \%$ & $8,2 \%$ & $1,6 \%$ & $1,6 \%$ & $100,0 \%$ \\
\hline 23-02-15 & 2013 & $32,9 \%$ & $23,3 \%$ & $19,2 \%$ & $6.8 \%$ & $6.8 \%$ & $-\%$ & $11,0 \%$ & $100,0 \%$ \\
\hline 23-02-15 & 2014 & $34,2 \%$ & $79 \%$ & $23,7 \%$ & $-\%$ & $15,8 \%$ & $5,3 \%$ & $13,2 \%$ & $100,0 \%$ \\
\hline & Total & $36,5 \%$ & $20,5 \%$ & $18,8 \%$ & $7,6 \%$ & $6,3 \%$ & $5,3 \%$ & $5,1 \%$ & $100,0 \%$ \\
\hline
\end{tabular}

\begin{tabular}{l|l|}
\hline Atualização & \\
\hline Quem atualiza? & $\begin{array}{l}\text { Pedir nova planilha de controle de estágios, contar o número de alunos por } \\
\text { indústria, incluir na tabela. Ter cuidado para não incluir entradas duplicadas. } \\
\text { (Classificação de indústria é feita manualmente, através de conhecimento prévio, } \\
\text { comparação com as entradas existentes, ou pesquisa) }\end{array}$ \\
\hline $\begin{array}{l}\text { Como atualiza? } \\
\text { Com que frequência? }\end{array}$ & Anualmente \\
\hline
\end{tabular}

\section{Targets and Benchmarks Não se aplicam - a não ser que se estabeleça uma meta \\ Metas \\ Comparativos}

Figura 1 - Reprodução da ficha do indicador Distribuição dos estágios por setor da economia.

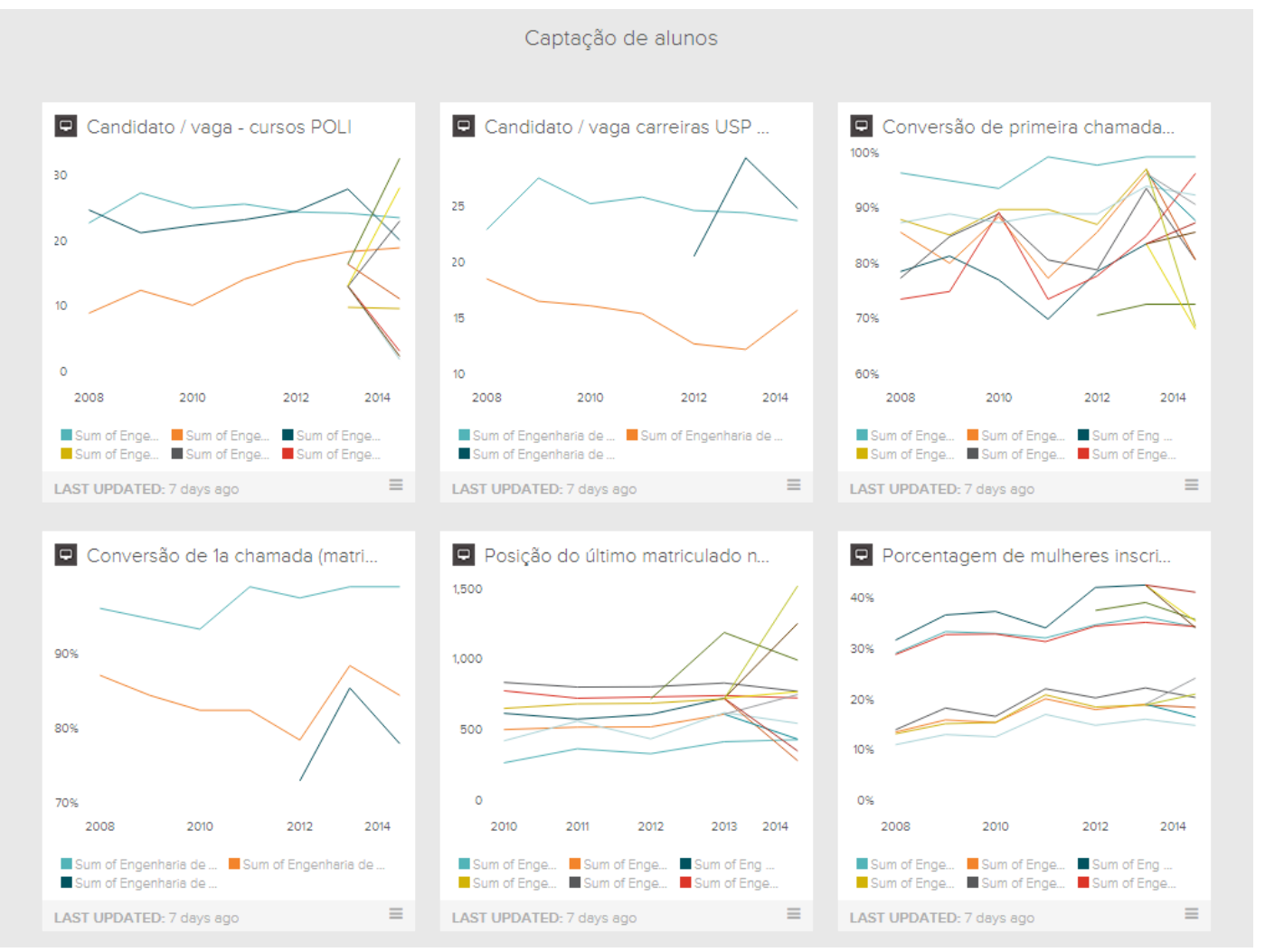

Figura 2 - Exemplo de tela do dashboard. Essa interface possibilita, por exemplo, a visualização simultânea de vários indicadores que compõem uma mesma perspectiva. 
Mais do que isso, pode ser útil determinar uma periodicidade (por exemplo, a cada três anos) na qual seja revisto e renovado o SMD em si, adicionando e removendo indicadores - e atualizando suas respectivas metas -, conforme os objetivos do departamento. Por fim, vale destacar que, com os gráficos e análises apresentados, já foi possível perceber diversas tendências entre os potenciais e atuais alunos do curso avaliado. Dentre elas, a queda da procura do curso, a redução lenta da desigualdade de gênero (em termos de números de alunos), o crescimento do estágio em empresas mais novas, com menos de dez anos de existência, como startups, e também a preferência pelo estágio em companhias de natureza menos técnica, como empresas de serviços e comércio (geralmente eletrônico).

Como limitações dessa proposta de SMD, destacam-se duas questões principais: primeiramente, seria interessante que os dados fornecidos pelas secretarias tivessem um nível maior de padronização, concisão e facilidade de compartilhamento. Para a maioria dos indicadores criados a partir desses conjuntos de dados, foi grande a necessidade de trabalho de padronização de dados - por exemplo, dos nomes das engenharias, e a eliminação de dados duplicados, entre outros. Em segundo lugar, muitos dos indicadores listados não puderam ser propriamente desenvolvidos pela falta de dados disponíveis.

\section{Conclusões}

Neste artigo foi apresentada uma proposta de sistema de indicadores de desempenho para a graduação em Engenharia aplicada ao curso de Engenharia de Produção da Escola Politécnica da Universidade de São Paulo.

O trabalho buscou fornecer uma abordagem para a gestão baseada em números no ensino superior, com aplicação em curso de graduação, especificamente para a graduação em Engenharia de Produção. Por meio da definição de um formato de SMD aplicável na educação superior, da sintetização de um processo de desenvolvimento de indicadores, da listagem de requisitos operacionais para indicadores, da seleção de indicadores de desempenho (coerentes com a aplicação proposta) presentes na literatura, da definição de um formato adequado de ficha de indicadores, e da escolha de ferramentas adequadas para a confecção de um painel de navegação (dashboard), obteve-se como resultado uma aplicação piloto, útil para o curso em questão, mas que também pode servir de referência para outros cursos.

Relembrando o que disse o ex-presidente do CNPq, Prof. Hernan Chaimovich: "[...] para aumentar aquilo que você chamou de qualidade da educação, a primeira coisa a fazer é medi-la" (CHAIMOVICH, 2015). Dessa forma, ainda que seja relativamente trabalhoso estruturar a gestão baseada em números, acredita-se que esse seja o caminho para a melhoria contínua na qualidade da educação oferecida.

\section{Referências Bibliográficas}

ALEXANDER, F. K. "The Changing Face of Accountability: Monitoring and Assessing Institutional Performance in Higher Education". The Journal of Higher Education, vol. 71, n. 4, 2000, pp. 411-431.

ARAUJO, A. O. Contribuição ao Estudo de Indicadores Desempenho de Empreendimentos Hoteleiros, sob o Enfoque da Gestão Estratégica. São Paulo, SP, Brasil: Faculdade de Economia, Administração e Contabilidade da Universidade de São Paulo, 2001.

BEARD, D. F. "Successful Applications of the Balanced Scorecard in Higher Education". Journal of Education for Business, maio/jun. 2009, pp. 275-282.

BORGES, J. G. \& CARVALHO, M. M. "Sistemas de Indicadores de Desempenho em Projetos". Revista de Gestão e Projetos - GeP, vol. 2, n. 1, jan.jjul. 2011, pp. 174-207.

CHAIMOVICH, H. "Novo presidente do CNPq, Chaimovich fala sobre pesquisa e desenvolvimento" (P. HEBMULLER, Entrevistador). Jornal da USP, 6 mar. 2015. Disponível em: <http://www5.usp.br/87545/novo-presidente-do-cnpq-chaimovich-fala-sobre-pesquisa -e-desenvolvimento/>. Acessado em 25 mar. 2015.

GHEN, S.-H.; YANG, C.-C. \& SHIAU, J.-Y. "The Application of Balanced Scorecard in the Performance Evaluation of Higher Education". The TQM Magazine, vol. 18, n. 2, 2006, pp. 190-205.

EWELL, P. "Developing Statewide Performance Indicators for Higher Education". In: RUPPERT, S. S. Charting Higher Education Accountability: A Sourcebook on State-Level Performance Indicators. Denver: Education Commission of the States, 1994. 
FRANCISCHINI, P. G. Indicadores de Desempenho I e II - Aulas 1 a 4. PRO2421 - Técnicas de Gerenciamento de Operações Industriais. São Paulo, SP, Brasil: Departamento de Engenharia de Produção da Escola Politécnica da USP, 2014.

FUVEST. Estatísticas, 2015. Disponível em: <http:// www.fuvest.br/vest2015/estat/estat.stm>. Acessado em 4 maio 2015.

KAPLAN, R. \& NORTON, D. P. The Balanced Scorecard. Boston, MA: Harvard Business Review Press, 1996.

KIYAN, F. M. Proposta para Desenvolvimento de Indicadores de Desempenho como Suporte Estratégico. São Carlos, SP, Brasil: Escola de Engenharia de São Carlos da Universidade de São Paulo, 2001.

MACHADO, A. G. "A Inadequação dos Sistemas Tradicionais de Medição de Desempenho diante do Novo Cenário Competitivo". Encontro Nacional de
Engenharia de Produção, Abepro, 2001.

MARTIN, M.; SAUVAGEOT, C. \& TCHATCHOUA, B. Constructing an Indicator System or Scorecard for Higher Education - A Practical Guide. Paris: International Institute for Educational Planning - Unesco, 2011.

ROSA, E.; PAMPLONA, E. \& ALMEIDA, D. "Parâmetros de Desempenho e a Competitividade dos Sistemas de Manufatura". Encontro Nacional de Engenharia de Produção (Enegep). São Carlos: ABEPRO/UFSCAR, 1995, pp. 519-522.

SPINOLA, M. \& PESSÔA, M. "Tecnologia da Informação". In: CONTADOR, J. C. Gestão de Operações: a Engenharia de Produção a Serviço da Modernização da Empresa. São Paulo: Editora Edgard Blucher, 1997, pp. 97-106.

THOMSON REUTERS. Finding Meaningful Performance Measures for Higher Education - A Report for Executives. Thomson Reuters, 2010.

Publicado em 04/07/2018. 